\title{
REGULAR BERNSTEIN BLOCKS
}

\author{
JEFFREY D. ADLER AND MANISH MISHRA
}

\begin{abstract}
For a connected reductive group $G$ defined over a non-archimedean local field $F$, we consider the Bernstein blocks in the category of smooth representations of $G(F)$. Bernstein blocks whose cuspidal support involves a regular supercuspidal representation are called regular Bernstein blocks. Most Bernstein blocks are regular when the residual characteristic of $F$ is not too small. Under mild hypotheses on the residual characteristic, we show that the Bernstein center of a regular Bernstein block of $G(F)$ is isomorphic to the Bernstein center of a regular depth-zero Bernstein block of $G^{0}(F)$, where $G^{0}$ is a certain twisted Levi subgroup of $G$. In some cases, we show that the blocks themselves are equivalent, and as a consequence we prove the ABPS Conjecture in some new cases.
\end{abstract}

\section{INTRODUCTION}

1.1. The problem. Let $F$ be a nonarchimedean local field of residual characteristic $p$, and $G$ a connected reductive $F$-group. One wants to understand the category $\mathcal{R}(G(F))$ of smooth, complex representations of $G(F)$. The Bernstein decomposition [5] allows us to decompose this category into a product $\prod_{\left\{[M, \sigma]_{G}\right\}} \mathcal{R}^{[M, \sigma]_{G}}(G(F))$ of full subcategories $\mathcal{R}^{[M, \sigma]_{G}}(G(F)$ ), called Bernstein blocks, so it is in some sense enough to understand each block. (Here, $[M, \sigma]_{G}$ ranges over the set of inertial classes of $G(F)$. Such a class is represented by an $F$-Levi subgroup $M$ of $G$ and an irreducible, supercuspidal representation $\sigma$ of $M(F)$, and consists of the set of all pairs $\left(M^{\prime}(F), \sigma^{\prime}\right)$ that are equivalent to $(M(F), \sigma)$ under twisting by unramified characters of $M(F)$ and conjugation by elements of $G(F)$.) Each block $\mathcal{R}^{[M, \sigma]_{G}}(G(F))$ is a module category over an algebra, and one can construct an appropriate Hecke algebra in a more-or-less explicit way if $[M, \sigma]_{G}$ has an associated Bushnell-Kutzko type [7]. In particular, if $\sigma$ has depth zero, then the structure of the Hecke algebra is given by Morris [19. Thus, in principle, one way to understand the category $\mathcal{R}(G(F))$ would be to show that for each block $\mathcal{R}^{[M, \sigma]_{G}}(G(F))$, there is another connected reductive $F$-group $G^{0}$ and a depth-zero block $\left[M^{0}, \sigma^{0}\right]_{G^{0}}$ for $G^{0}(F)$ such that the blocks are equivalent as categories.

Our aim in this paper is to study the structure of Bernstein blocks using the above approach in many cases.

Let us remark that Kim and $\mathrm{Yu}$ [17, give a construction of types (see [17, §7.4] for more details), and Fintzen [9] has recently shown that if $G$ splits over a tame extension of $F$ and $p$ does not divide the order of the Weyl group of $G$, then every Bernstein block for $G(F)$ admits a type arising from this construction. The Kim-Yu construction starts with a certain datum $\Sigma$, out of which it produces a

2020 Mathematics Subject Classification. 22E50.

The second named author was partially supported by SERB MATRICS and SERB ERCA grants. 
sequence of types $\left(K^{i}, \rho_{i}\right)$ for groups $G^{i}(F), 0 \leq i \leq d$, where $\vec{G}=\left(G^{0} \subsetneq \ldots \subsetneq\right.$ $\left.G^{d}=G\right)$ is a tower of twisted Levi subgroups of $G$ and which is part of the datum $\Sigma$. Write $(K, \rho)$ for $\left(K^{d}, \rho_{d}\right)$ and write $\mathcal{H}\left(G, \rho^{\vee}\right)$ (resp. $\left.\mathcal{H}\left(G^{0}, \rho_{0}^{\vee}\right)\right)$ for the Hecke algebra associated to the type $(K, \rho)$ (resp. $\left.\left(K^{0}, \rho_{0}\right)\right)$. Denote by $\mathcal{R}_{\mathrm{f}}(G, \rho)$ (resp. $\left.\mathcal{R}_{\mathrm{f}}\left(G^{0}, \rho_{0}\right)\right)$, the category of finite-length modules over $\mathcal{H}\left(G, \rho^{\vee}\right)\left(\operatorname{resp} . \mathcal{H}\left(G^{0}, \rho_{0}^{\vee}\right)\right)$. Existing results [12,14,16,20,21], together with a conjecture of $Y u$ [26, Conjecture $0.2])$ on supercuspidal blocks, suggest a more general conjecture:

Conjecture 1.1. There exists a bijection, determined by the choice of $\Sigma$, between the simple modules of $\mathcal{H}\left(G, \rho^{\vee}\right)$ and those of $\mathcal{H}\left(G^{0}, \rho_{0}^{\vee}\right)$.

While the Hecke algebra $\mathcal{H}\left(G^{0}, \rho_{0}^{\vee}\right)$ is determined up to isomorphism, it (and thus the bijection of the Conjecture) depends on $\Sigma$, which is not uniquely determined.

Remark 1.2. In many cases, something stronger than Conjecture 1.1 holds. Namely, the datum $\Sigma$ determines an isomorphism of Hecke algebras

$$
\mathcal{H}\left(G, \rho^{\vee}\right) \stackrel{\sim}{\longrightarrow} \mathcal{H}\left(G^{0}, \rho_{0}^{\vee}\right) .
$$

This is however not true in general. In many cases where the isomorphism of Hecke algebras fails, it may still be true that there exists an equivalence of categories

$$
\mathcal{R}_{\mathrm{f}}(G, \rho) \cong \mathcal{R}_{\mathrm{f}}\left(G^{0}, \rho_{0}\right),
$$

determined by the choice of $\Sigma$.

1.2. Bernstein center. In [15, Kaletha studies a large class of superpercuspidal representations which he calls regular. Most supercuspidal representations are of this kind when $p$ is not too small (see the paragraph following [15, Definition 3.7.3] to understand what is meant by "most"). We call a Bernstein block $\mathcal{R}^{[M, \sigma]_{G}}(G(F))$ regular if $\sigma$ is a regular supercuspidal representation. Under certain restrictions on $p$, we show in Theorem 7.4 that

Theorem 1.5. The Bernstein center of a regular Bernstein block $\mathcal{R}^{[M, \sigma]_{G}}(G(F))$ is isomorphic to the Bernstein center of a depth-zero regular Bernstein block $\mathcal{R}^{\left[M^{0}, \sigma_{0}\right]_{G^{0}}}\left(G^{0}(F)\right)$ of a twisted Levi subgroup $G^{0}$ of $G$.

When $M=G$, this result is covered by [18, Theorem 6.1] which proves such an isomorphism for tame supercuspidal blocks. Thus, Theorem 1.5 is a generalization for regular Bernstein blocks of the main result of [18].

1.3. Hecke algebra isomorphism. Suppose $M=G$. Then Conjecture 1.1 would follow from [26, Conjecture 0.2], which appears here as Conjecture 6.3. We show (Corollary 6.4) that the conjecture is true if the $F$-split rank of the center of $G$ is at most 1; or if $\sigma$ is generic; or if $p$ satisfies some mild hypotheses, and the restriction of $\sigma$ to the derived group of $G$ decomposes into regular supercuspidal representations.

Now let us consider the case where $M$ is not necessarily equal to $G$. Let $(K, \rho)$ be the $[M, \sigma]_{G}$-type obtained out of the datum $\Sigma$ by the Kim-Yu construction. Assume that the tower $\vec{G}$ of twisted Levi subgroups contained in the datum $\Sigma$ consists of $F$-Levi subgroups. Then we show (in Corollary 8.6) that Equation (1.3) and consequently Conjecture 1.1 holds. This follows as an easy consequence of the results in [17. The condition that the tower $\vec{G}$ consists of $F$-Levi subgroups holds for instance when the connected center of $G^{0}$ is split modulo the center of $G$. In 
particular, it holds when $G$ is split and $M$ is a maximal split torus in $G$. Thus, as a very special case, one obtains Equation (1.3) (and consequently Conjecture 1.1) for principal series blocks of split groups, recovering the result of Roche 22. We also prove Equation (1.3) for certain principal series blocks of non-split groups (Proposition 8.4) for suitably large $p$.

We prove weaker versions of Equation (1.3) for more general situations in $\$ 10$,

1.4. Consequences for the ABPS Conjecture. Aubert, Baum, Plymen, and Solleveld [3] conjecture that the set of irreducible objects in a Bernstein block has the structure of a particular twisted extended quotient. This "ABPS Conjecture" holds for depth-zero Bernstein blocks due to the work of Solleveld [25]. We show, using this result of Solleveld, that under certain restrictions on $p$, Conjecture 1.1 for a regular Bernstein block implies ABPS Conjecture for that block. Consequently, we obtain the ABPS Conjecture for many new cases (Theorem 9.5), namely those mentioned in the previous section. In particular, as the simplest case, we obtain ABPS Conjecture for principal series blocks of split groups. This reproves a part of the main result in [4] in a much shorter way. However, unlike Theorem 9.5, the proof in loc. cit. is independent of the main result of [25].

Solleveld has, in a recent preprint, given a complete proof of the ABPS Conjecture [24, Theorem D]. His proof bypasses the type-theoretic approach and builds instead on the ideas of Bernstein [5] and Heiermann [13. Moreover, under certain technical hypotheses (the existence and compatibility of certain co-cycles), he also shows [24, Theorem B] that the category $\mathcal{R}_{\mathrm{f}}(G, \rho)$ is equivalent to the finite-length module category of a twisted affine Hecke algebra. Under the same hypothesis and some additional residue characteristic hypotheses (Hypothesis 5.1), his results may be used to establish Equation (1.4) for regular Bernstein blocks provided one shows that the isomorphisms of Theorems 7.1 and 7.3 respect a certain finer structure dictated by Harish-Chandra $\mu$-function. We plan to pursue these matters in a subsequent work.

1.5. Acknowledgments. It is a pleasure for the authors to thank the following. Maarten Solleveld clarified for us the definition of the twisted extended quotient and pointed out an error in a previous draft. Anne-Marie Aubert pointed out that the isomorphism of Hecke algebras in Remark 1.2 is false in general for tame Bernstein blocks. Adéle Bourgeois pointed out a typographical error in Definition 5.2 An anonymous referee provided comments that helped us to improve the exposition of this work.

\section{Notation}

Throughout this article, $F$ denotes a non-archimedean local field of residue characteristic $p$. If $G$ is a reductive $F$-group and $K$ is a subgroup of $G(F)$, we will denote $g K g^{-1}$ by ${ }^{g} K$ for $g \in G(F)$. If $\rho$ is a representation of $K$, then ${ }^{g} \rho$ will denote the representation $x \mapsto \rho\left(g^{-1} x g\right)$ of ${ }^{g} K$. If $H$ is a subgroup of a group $G$, then $N_{G}(H)$ denotes the normalizer of $H$ in $G$.

Write $\mathcal{B}(G, F)$ for the Bruhat-Tits building of $G(F)$. For any point $x \in \mathcal{B}(G, F)$, we write $G(F)_{x, 0}$ for the parahoric subgroup of $G(F)$ associated to $x$, and we let $G(F)_{x: 0: 0+}$ denote the quotient of $G(F)_{x, 0}$ by its maximal pro- $p$ subgroup. If $G$ is a torus, then we omit $x$ from the notation and just write $G(F)_{0}$ for the unique 
parahoric subgroup of $G(F)$, and we also let $G(F)_{\mathrm{b}}$ denote the unique maximal bounded subgroup.

For an $F$-group $A$ whose connected part is a torus, let $A_{\mathrm{s}}$ and $A_{\text {an }}$ denote the maximal $F$-split and $F$-anisotropic subtori in $A$, respectively.

\section{Review of the Bernstein CEnter}

Throughout, $G$ denotes a connected reductive $F$-group. We quickly review general theory and fix some notation.

3.1. Bernstein decomposition. Let ${ }^{\circ} G(F)$ denote the subgroup

$$
\left\{g \in G(F):|\nu(g)|=1 \text { for every character } \nu \in \operatorname{Hom}_{F}\left(G, \mathbb{G}_{\mathrm{m}}\right)\right\},
$$

and let $X_{\mathrm{nr}}(G(F))=\operatorname{Hom}\left(G(F) /{ }^{\circ} G(F), \mathbb{C}^{\times}\right)$. The group $X_{\mathrm{nr}}(G(F))$ is called the group of unramified characters of $G(F)$. Consider the pairs $(L(F), \sigma)$ where $L$ is an $F$-Levi subgroup of $G$ and $\sigma$ is an irreducible supercuspidal representation of $L(F)$. Denote by $[L, \sigma]_{G}$ its inertial equivalence class: the set of pairs $\left(L^{\prime}, \sigma^{\prime}\right)$, such that $L^{\prime}$ is an $F$-Levi subgroup of $G, \sigma^{\prime}$ is an irreducible, supercuspidal representation of $L(F)$, and $\left(L^{\prime}, \sigma^{\prime}\right)=\left({ }^{g} L,{ }^{g} \sigma \otimes \nu\right)$ for some $g \in G(F)$ and $\nu \in X_{\mathrm{nr}}(L(F))$. The set of inertial equivalence classes is called the Bernstein spectrum of $G(F)$, which we denote by $\mathfrak{B}(G, F)$. Let $\mathcal{R}(G(F))$ denote the category of smooth representations of $G(F)$. We say that a smooth irreducible representation $\pi$ has inertial support $\mathfrak{s}=[L, \sigma]_{G}$ if $\pi$ appears as a subquotient of a representation parabolically induced from some element of the class $\mathfrak{s}$. Define a full subcategory $\mathcal{R}^{\mathfrak{s}}(G(F))$ of $\mathcal{R}(G(F))$ as follows: $\pi \in \mathcal{R}(G(F))$ belongs to $\mathcal{R}^{\mathfrak{s}}(G(F))$ if each irreducible subquotient of $\pi$ has inertial support $\mathfrak{s}$. We will denote the class of irreducible objects in $\mathcal{R}^{\mathfrak{s}}(G(F))$ by $\operatorname{Irr}^{\mathfrak{s}}(G(F))$.

Theorem 3.1 (Bernstein). We have a decomposition

$$
\mathcal{R}(G(F))=\prod_{\mathfrak{s} \in \mathfrak{B}(G, F)} \mathcal{R}^{\mathfrak{s}}(G(F)) .
$$

3.2. Hecke algebra. Let $(\tau, W)$ be an irreducible representation of a compact open subgroup $J$ of $G(F)$. The Hecke algebra $\mathcal{H}(G, \tau)$ is the space of compactly supported functions $f: G(F) \longrightarrow \operatorname{End}_{\mathbb{C}}(\tau)$ satisfying

$$
f\left(j_{1} g j_{2}\right)=\tau\left(j_{1}\right) f(g) \tau\left(j_{2}\right) .
$$

The standard convolution algebra gives $\mathcal{H}(G, \tau)$ the structure of an associative $\mathbb{C}$-algebra with identity.

We say that $(J, \tau)$ is an $\mathfrak{s}$-type if $\mathcal{R}^{\mathfrak{s}}(G(F))$ is precisely the subcategory of $\mathcal{R}(G(F))$ consisting of smooth representations which are generated by their $\tau$ isotypic component. In that case, $\mathcal{R}^{\mathfrak{s}}(G(F))$ is equivalent to the category of nondegenerate modules of $\mathcal{H}\left(G, \tau^{\vee}\right)$, where $\tau^{\vee}$ is the dual of $\tau$ ([7]).

3.3. Bernstein center. Let $\mathfrak{s}=[L, \sigma]_{G} \in \mathfrak{B}(G, F)$ and set $\mathfrak{t}=[L, \sigma]_{L} \in \mathfrak{B}(L, F)$. The center $\mathfrak{Z}(G(F))$ (resp. $\mathfrak{Z}^{\mathfrak{s}}(G(F))$, resp. $\mathfrak{Z}^{\mathfrak{t}}(L(F))$ ) of the category $\mathcal{R}(G(F))$ (resp. $\mathcal{R}^{\mathfrak{s}}(G(F))$, resp. $\left.\mathcal{R}^{\mathfrak{t}}(L(F))\right)$ is called its Bernstein center. Recall here that the center of an abelian category is the endomorphism ring of the identity functor. Let

$$
N^{\mathfrak{s}}=\left\{n \in N_{G}(L)(F) \mid{ }^{n} \sigma \cong \sigma \nu \text { for some } \nu \in X_{\mathrm{nr}}(L(F))\right\},
$$


and write $W^{\mathfrak{s}}=N^{\mathfrak{s}} / L(F)$. The set $\operatorname{Irr}^{\mathfrak{t}}(L(F))$ of irreducible objects of $\mathcal{R}^{\mathfrak{t}}(L(F))$ has the structure of a complex affine variety, on which the group $W^{\mathfrak{s}}$ acts algebraically. (The variety $\operatorname{Irr}^{\mathrm{t}}(L(F))$ is (non-canonically) isomorphic to the quotient $X_{\mathrm{nr}}(L(F)) /\left\{\chi \in X_{\mathrm{nr}}(L(F)) \mid \sigma \chi=\sigma\right\}$.)

Theorem 3.2 (Bernstein). [5] The Bernstein center of $\mathcal{R}^{\mathfrak{s}}(G(F))$ can be viewed as the ring of regular functions on the quotient variety $\operatorname{Irr}^{\mathfrak{t}}(L(F)) / W^{\mathfrak{s}}$.

\section{REVIEW OF TAME AND REGULAR SUPERCUSPIDAL REPRESENTATIONS}

4.1. Tame supercuspidal representations. Assume that $G$ splits over the maximal tamely ramified extension $F^{\mathrm{t}}$ of $F$. A tamely twisted Levi subgroup of $G^{\prime}$ of $G$ is a reductive $F$-subgroup such that $G^{\prime} \otimes F^{\mathrm{t}}$ is an $F^{\mathrm{t}}$-Levi subgroup of $G \otimes F^{\mathrm{t}}$. Let $\Sigma$ denote the datum $\left(\vec{G}, \pi_{0}, \vec{\phi}\right)$, where $\vec{G}=\left(G^{0}, \ldots, G^{d}\right)$ is a tower of algebraic subgroups of $G$,

$$
G^{0} \subsetneq \ldots \subsetneq G^{d}=G,
$$

such that $Z\left(G^{0}\right) / Z(G)$ is anisotropic and each $G^{i}$ is a tamely twisted Levi subgroup of $G, \pi_{0}$ is a depth-zero supercuspidal representation of $G^{0}(F)$ and $\vec{\phi}=\left(\phi_{0}, \ldots, \phi_{d}\right)$ is such that $\phi_{i}: G^{i}(F) \longrightarrow \mathbb{C}^{\times}$is a smooth character of depth $r_{i}>0$. We require the datum $\Sigma$ to satisfy several technical conditions. (See [11, §3.1] for a precise list. Note that this source and others, e.g., [15], use the notation $\pi_{-1}$ for the representation that we are calling $\pi_{0}$.)

Yu's construction [26] produces a supercuspidal representation $\pi$ out of the datum $\Sigma$. Recently, Fintzen [10] has shown that if the residue characteristic $p$ does not divide the order of the Weyl group, this construction yields all supercuspidal representations of $G(F)$.

4.2. Regular supercuspidal representations. Let $(S, \theta)$ be a pair consisting of tame maximal $F$-torus $S$ in $G$ and a character $\theta: S(F) \longrightarrow \mathbb{C}^{\times}$. A Kaletha-Howe factorization of $(S, \theta)$ is a pair $(\vec{G}, \vec{\phi})$, where $\vec{G}=\left(S=G^{-1} \subset G^{0} \subsetneq \ldots \subsetneq G^{d}=G\right)$ is a tower of tamely twisted Levi $F$-subgroups and $\vec{\phi}=\left(\phi_{-1}, \ldots, \phi_{d}\right)$ is a sequence of characters $\phi_{i}: G^{i}(F) \longrightarrow \mathbb{C}^{\times}$and satisfying

$$
\theta=\left.\prod_{i=-1}^{d} \phi_{i}\right|_{S(F)}
$$

and some additional technical conditions (see [15, Definition 3.6.2]).

If the datum $(S, \theta)$ is a tame regular elliptic pair, i.e., it satisfies the conditions in [15. Definition 3.7.5], then the pair $\left(S, \phi_{-1}\right)$ determines a depth-zero supercuspidal representation $\pi_{0}$ of $G^{0}(F)$ and the datum

$$
\Sigma=\left(\left(G^{0} \subsetneq \ldots \subsetneq G^{d}=G\right), \pi_{0},\left(\phi_{0}, \ldots, \phi_{d}\right)\right)
$$

is a $\mathrm{Yu}$ datum and therefore produces a supercuspidal representation $\pi(S, \theta)$ of $G(F)$. These representations are called regular supercuspidal representations.

If $(S, \theta)$ and $\left(S^{\prime}, \theta^{\prime}\right)$ are tame regular elliptic pairs in $G$, then $\pi(S, \theta)$ and $\pi\left(S^{\prime}, \theta^{\prime}\right)$ are equivalent if and only if $(S, \theta)$ and $\left(S^{\prime}, \theta^{\prime}\right)$ are conjugate in $G(F)$. 


\section{A RESUlt ON MULTIPLICITY ONE UPON RESTRICTION}

In this section, we will assume the following.

Hypothesis 5.1. The residue characteristic $p$ of $F$ is not a bad prime for $G$ (see [15, §2.1]) and does not divide the order of the fundamental group of the derived group $G^{\text {der }}$ of $G$.

Let $(S, \theta)$ be a tame regular elliptic pair in $G$ and $\pi(S, \theta)$ the associated regular supercuspidal representation. Let $G^{\prime}$ be an $F$-subgroup of $G$ such that $G^{\text {der }} \subset$ $G^{\prime} \subset G$. Write $S^{\prime}=S \cap G^{\prime}$ and $\theta^{\prime}=\left.\theta\right|_{S^{\prime}(F)}$. Let $N_{G(F)}\left(S^{\prime},\left.\theta^{\prime}\right|_{S^{\prime}(F)_{0}}\right)$ denote the stabilizer of $\left.\theta^{\prime}\right|_{S^{\prime}(F)_{0}}$ in $N_{G}\left(S^{\prime}\right)(F)$.

Definition 5.2. The pair $(S, \theta)$ is regular in $G^{\prime}$ if $N_{G(F)}\left(S^{\prime},\left.\theta^{\prime}\right|_{S^{\prime}(F)_{0}}\right)=S(F)$.

Theorem 5.3. If $(S, \theta)$ is a tame regular elliptic pair in $G$ which is regular in $G^{\prime}$, then $\left.\pi(S, \theta)\right|_{G^{\prime}(F)}$ is multiplicity free.

We observe that $(S, \theta)$ is regular in $G^{\prime}$ if and only if the components of $\left.\pi\right|_{G^{\prime}(F)}$ are regular supercuspidals. If $(S, \theta)$ is not regular in $G^{\prime}$, then higher multiplicities can occur, even though $(S, \theta)$ is regular in $G$. See [2, $\S 7]$ for an example.

Looking at general irreducible representations of $G(F)$, there are many situations where one can prove that the restriction to $G^{\prime}(F)$ is multiplicity free. See [8] for a conjecture, together with a proof for tempered representation under some assumptions about the local Langlands correspondence. See [2] for a conjecture, reduction to the tempered case, examples, and an announcement of Theorem 5.3 .

Proof of Theorem 5.3. Since $(S, \theta)$ is regular in $G^{\prime}$, it follows from [15, Definition 3.7.5 and Lemma 3.6.5] that $\left(S^{\prime}, \theta^{\prime}\right)$ is a tame regular elliptic pair in $G^{\prime}$. Let $V^{\prime}$ (resp. $V$ ) be the space realizing the representation $\pi\left(S^{\prime}, \theta^{\prime}\right)$ (resp. $\left.\pi(S, \theta)\right)$. One can describe these spaces explicitly, since each regular supercuspidal representation is induced from a representation of a compact open subgroup. We now describe an embedding of $V^{\prime}$ in $V$.

From Yu's construction [26], we see that

- $\pi(S, \theta)$ is induced from a smooth irreducible representation $\rho$ of an open subgroup $K$ of $G(F)$ that is compact modulo the center of $G(F)$;

- $\pi\left(S^{\prime}, \theta^{\prime}\right)$ is similarly induced from $\left(K^{\prime}, \rho^{\prime}\right)$; and

- we can realize $\rho^{\prime}$ as an irreducible subrepresentation of $\left.\rho\right|_{K^{\prime}}$.

Let $W$ and $W^{\prime}$ be the spaces realizing $\rho$ and $\rho^{\prime}$. Thus, $W^{\prime}$ is a subspace of $W$. We have

$$
W=\bigoplus_{k \in K / \operatorname{Stab}_{K}\left(W^{\prime}\right)}{ }^{k} W^{\prime} .
$$

Pick coset representatives $k_{1}, \ldots, k_{n}$ of $K / \operatorname{Stab}_{K} W^{\prime}$. Let

$$
\iota: \operatorname{End}\left(W^{\prime}\right) \longrightarrow \operatorname{End}(W)
$$

be given by

$$
\iota: \lambda^{\prime} \mapsto \bigoplus_{i=1}^{n} k_{i} \lambda^{\prime}
$$


Then for all $k^{\prime} \in K^{\prime}, \iota\left(\rho^{\prime}\left(k^{\prime}\right)\right)=\rho\left(k^{\prime}\right)$. Define an embedding $\iota: V^{\prime} \longrightarrow V$ by setting, for $f^{\prime} \in V^{\prime}, \iota\left(f^{\prime}\right)$ to be the function $f \in V$ given by

$$
f(g)=\left\{\begin{array}{ll}
0 & \text { if } g \notin G^{\prime}(F) K, \\
\iota\left(f^{\prime}\left(g^{\prime}\right)\right) \rho(k) & \text { if } f=g^{\prime} k, \text { where } g^{\prime} \in G^{\prime}(F), k \in K
\end{array} .\right.
$$

To see that the function $f=\iota\left(f^{\prime}\right)$ is well defined, let $g_{1}^{\prime} k_{1}=g_{2}^{\prime} k_{2}$, where $g_{1}^{\prime}, g_{2}^{\prime} \in$ $G^{\prime}(F)$ and $k_{1}, k_{2} \in K$. Then $g_{1}^{\prime-1} g_{2}^{\prime}=k_{1} k_{2}^{-1} \in G^{\prime}(F) \cap K=K^{\prime}$. We have

$$
\begin{aligned}
\iota\left(f^{\prime}\left(g_{2}^{\prime}\right) \rho\left(k_{2}\right)\right. & =\iota\left(f^{\prime}\left(g_{1}^{\prime} g_{1}^{\prime-1} g_{2}^{\prime}\right) \rho\left(k_{2}\right)\right. \\
& =\iota\left(f^{\prime}\left(g_{1}^{\prime}\right)\right) \rho\left(k_{1} k_{2}^{-1}\right) \rho\left(k_{2}\right) \\
& =\iota\left(f^{\prime}\left(g_{1}^{\prime}\right)\right) \rho\left(k_{1}\right) .
\end{aligned}
$$

This proves that $f$ is well defined. Now since $S(F) \subset K$, it follows that $S(F)$ preserves $\iota\left(V^{\prime}\right)$. Thus

$$
\operatorname{Stab}_{G(F)} \iota\left(V^{\prime}\right) \supseteq S(F) G^{\prime}(F) .
$$

By Clifford theory

$$
\pi(S, \theta)=\bigoplus_{g \in G(F) / \operatorname{Stab}_{G(F)} \iota\left(V^{\prime}\right)}{ }^{g} \pi\left(S^{\prime}, \theta^{\prime}\right) .
$$

We claim that for $g \notin \operatorname{Stab}_{G(F)} \iota\left(V^{\prime}\right),{ }^{g} \pi\left(S^{\prime}, \theta^{\prime}\right) \not \pi\left(S^{\prime}, \theta^{\prime}\right)$. For suppose that ${ }^{g} \pi\left(S^{\prime}, \theta^{\prime}\right) \cong \pi\left(S^{\prime}, \theta^{\prime}\right)$ for some $g \in G(F)$. Equivalently, $\left({ }^{g} S^{\prime},{ }^{g} \theta^{\prime}\right)$ is $G^{\prime}(F)$-conjugate to $\left(S^{\prime}, \theta^{\prime}\right)$. So there exists a $g^{\prime} \in G^{\prime}(F)$ such that $g^{\prime} g$ stabilizes $\left(S^{\prime}, \theta^{\prime}\right)$. So $g \in$ $G^{\prime}(F) N_{G(F)}\left(S^{\prime}, \theta^{\prime}\right)$. But by hypothesis, $N_{G(F)}\left(S^{\prime},\left.\theta^{\prime}\right|_{S^{\prime}(F)_{0}}\right) \subset S(F)$. It follows that $g \in \operatorname{Stab}_{G(F)} \iota\left(V^{\prime}\right)$. This proves the claim.

Thus the summands ${ }^{g} \pi\left(S^{\prime}, \theta^{\prime}\right)$ for $g \in G(F) / \operatorname{Stab}_{G(F)} \iota\left(V^{\prime}\right)$ are non-isomorphic and consequently, $\left.\pi(S, \theta)\right|_{G^{\prime}(F)}$ is multiplicity free.

\section{HeCKe AlgEBRA FOR SUPERCUSPIDAL BLOCKS}

Let $(\pi, V)$ denote a supercuspidal representation of $G(F)$. Then $\left.\pi\right|_{\circ} G(F)$ is a finite direct sum of finite representations of ${ }^{\circ} G(F)$, all of which are $G(F)$-conjugate up to isomorphism. Fix an irreducible component $\left({ }^{\circ} \pi,{ }^{\circ} V\right)$ of $\left.\pi\right|_{\circ} G(F)$. Then $\operatorname{End}_{G(F)}\left(\operatorname{ind}_{\circ}^{G(F)}{ }^{\circ}{ }^{\circ} \pi\right)$ is canonically isomorphic to the convolution algebra $\mathcal{H}\left(G,{ }^{\circ} \pi\right)$ of ${ }^{\circ} \pi$-spherical functions $([23, \S 1.1 .2])$ and the Bernstein component $\mathcal{R}^{\mathfrak{s}}(G(F))$ is canonically equivalent to the module category of $\mathcal{H}\left(G,{ }^{\circ} \pi^{\vee}\right)$, where $\mathfrak{s}=[G, \pi]_{G}$.

Theorem 6.1. 23, Prop. 1.6.1.2] The Hecke algebra $\mathcal{H}\left(G,{ }^{\circ} \pi\right)$ is commutative if and only if $\left.\pi\right|_{{ }_{\circ} G(F)}$ is multiplicity free.

Proposition 6.2. The restriction $\left.\pi\right|_{{ }^{\circ} G(F)}$ is multiplicity free if the split rank of the identity component of the center of $G$ is 0 or 1 .

Proof. Let $Z$ denote the center of $G$. Then in this situation, $G(F) /\left(Z(F) \cdot{ }^{\circ} G(F)\right)$ is cyclic. The result then follows from [23, Remark 1.6.1.3].

Now let $\pi$ be a tame supercuspidal representation of $G(F)$. Then by Yu's construction, $\pi$ is compactly induced from a representation $\rho$ of an open, compact mod center subgroup $K$ of $G(F)$. Let ${ }^{\circ} K$ denote the maximal compact subgroup $K \cap{ }^{\circ} G(F)$ of $K$, and let ${ }^{\circ} \rho$ be any irreducible component of $\rho{ }_{\circ} K$. Then $\left({ }^{\circ} K,{ }^{\circ} \rho\right)$ is an $\mathfrak{s}$-type where $\mathfrak{s}=[G, \pi]_{G}$ ([7, Prop. 5.4]). The representation $\pi$ is constructed out of a depth-zero representation $\pi_{0}$ of a twisted Levi subgroup $G^{0}$ of $G$ together 
with additional data. The representation $\pi_{0}$ is compactly induced from a representation $\rho_{0}$ of a compact mod center subgroup $K^{0}$ of $G^{0}(F)$. Define an $\mathfrak{s}_{0}$-type $\left({ }^{\circ} K^{0},{ }^{\circ} \rho_{0}\right)$ analogously, where $\mathfrak{s}_{0}=\left[G^{0}(F), \pi_{0}\right]_{G^{0}(F)}$.

Jiu-Kang Yu makes the following conjecture [26, Conjecture 0.2].

Conjecture 6.3 (Yu's conjecture). There is an algebra isomorphism

$$
\mathcal{H}\left(G,{ }^{\circ} \rho\right) \cong \mathcal{H}\left(G^{0},{ }^{\circ} \rho_{0}\right) .
$$

Corollary 6.4. Yu's conjecture holds if any of the following conditions hold:

(a) The split rank of the identity component of the center of $G$ is 0 or 1 .

(b) Hypothesis 5.1 holds, and the irreducible components of $\left.\pi\right|_{G^{\mathrm{der}}(F)}$ are regular.

(c) $\pi$ is generic.

Proof. By [18, Cor. 6.3], the centers of $\mathcal{H}\left(G,{ }^{\circ} \rho\right)$ and $\mathcal{H}\left(G^{0},{ }^{\circ} \rho_{0}\right)$ are isomorphic. Thus, the conjecture is true if both of these Hecke algebras are commutative. From Theorem 6.1 it will be enough to show that $\pi$ and $\pi_{0}$ restrict without multiplicity to ${ }^{\circ} G(F)$ and ${ }^{\circ} G^{0}(F)$, respectively. Suppose condition (a) holds. Then our restrictions are multiplicity free from Proposition 6.2. together with the fact that the centers of $G$ and $G^{0}$ have the same split rank. Suppose condition (b) holds. By [15, Lemma 3.6.5], the components of $\left.\pi_{0}\right|_{\left(G^{0} \cap G^{\mathrm{der}}\right)(F)}$ are regular. Thus our restrictions are multiplicity free from Theorem 5.3. Suppose condition (c) holds. From a result of Stephen DeBacker and Cheng-Chiang Tsai, $\pi_{0}$ is also generic, and so our restrictions are multiplicity free from [23, Remark 1.6.1.3].

Note that in proving that Yu's conjecture holds under condition (c), we have used a result that is not yet in the literature. Readers who are unhappy about this can strengthen condition (c) to assert that both $\pi$ and $\pi_{0}$ are generic.

\section{BERNSTEIN CENTER OF REGULAR BLOCKS}

Assume Hypothesis 5.1 .

Let $L$ be an $F$-Levi subgroup of $G$ and let $(S, \theta)$ be a tame regular elliptic pair in $L$. Write $\pi=\pi(S, \theta)$ for the associated regular supercuspidal representation of $L(F)$. The pair $(S, \theta)$ produces a chain $\left(S=G^{-1} \subseteq G^{0} \varsubsetneqq \ldots \varsubsetneqq G^{d}=G\right)$ of twisted Levi subgroups of $G$. Write $L^{0}=L \cap G^{0}$. Let $\phi_{i}: G^{i}(F) \longrightarrow \mathbb{C}^{\times},-1 \leq i \leq d$, be the sequence of characters obtained from a Howe factorization of $(S, \theta)$. Then $\theta=\theta_{-} \theta_{+}$, where $\theta_{-}=\left.\phi_{-1}\right|_{S(F)}$ and $\theta_{+}=\left.\prod_{i=0}^{d} \phi_{i}\right|_{S(F)}$. It follows from 15, Lemma $3.6 .5]$ that $\left(S, \theta_{-}\right)$is a depth-zero tame regular elliptic pair for $L^{0}$. Let $\pi_{0}\left(S, \theta_{-}\right)$ denote the associated depth-zero regular supercuspidal representation of $L^{0}(F)$.

Write $\mathfrak{t}=[L, \pi]_{L}$ and $\mathfrak{t}_{0}=\left[L^{0}, \pi_{0}\right]_{L^{0}}$.

Theorem 7.1. [18, Theorem 6.1] The map

$$
\mathfrak{f}: \pi \otimes \nu \in \operatorname{Irr}^{\mathfrak{t}}(L(F)) \mapsto \pi_{0} \otimes\left(\left.\nu\right|_{L^{0}(F)}\right) \in \operatorname{Irr}^{\mathrm{t}_{0}}\left(L^{0}(F)\right), \quad \nu \in X_{\mathrm{nr}}(L(F)),
$$

is an isomorphism of varieties.

For $\mathfrak{s}=[L, \pi]_{G}$, recall that we denote by $W^{\mathfrak{s}}$ the stabilizer of $\mathfrak{s}$ in $\left(N_{G} L\right)(F) / L(F)$, i.e., $W^{\mathfrak{s}}=N^{\mathfrak{s}} / L(F)$, where

$$
N^{\mathfrak{s}}=\left\{n \in N_{G}(L)(F) \mid{ }^{n} \pi \cong \pi \nu, \text { for some } \nu \in X_{\mathrm{nr}}(L(F))\right\} .
$$

Similarly define $\mathfrak{s}_{0}, N^{\mathfrak{s}_{0}}$ and $W^{\mathfrak{s}_{0}}$ by replacing $L, \pi$ and $G$ in the above definition by $L^{0}, \pi_{0}$ and $G^{0}$. 
Lemma 7.2. The stabilizer of $\left.\theta\right|_{S(F)_{\mathrm{b}}}$ in $N_{G}(S)(F)$ lies in $N_{G^{0}}(S)(F)$ and equals the stabilizer of $\left.\theta_{-}\right|_{S(F)_{\mathrm{b}}}$ there.

Proof. The proof is identical to the proof of [15, Lemma 3.6.5] when we replace $S(F)_{r}$ there with $S(F)_{\mathrm{b}}$.

We observe here that $\theta_{+}$is invariant under $N_{G}(S)(F)$. This follows from the observation in the first paragraph of the proof of [15, Lemma 3.6.5] that for $0 \leq$ $i \leq d,\left.\phi_{i}\right|_{S(F)}$ is invariant under $N_{G}(S)(F)$.

Theorem 7.3. There is a group isomorphism

$$
\iota_{\pi}: W^{\mathfrak{s}} \cong W^{\mathfrak{s}_{0}} .
$$

Moreover, the isomorphism $\mathfrak{f}$ is equivariant with respect to the isomorphism $\iota_{\pi}$.

We remark here that since $G^{0}$ depends on the choice of $(S, \theta)$, so does the isomorphism $\iota_{\pi}$.

Proof. Take $n \in N^{\mathfrak{s}}$. So $n \in N_{G}(L)(F)$ and ${ }^{n}(S, \theta)$ is $L(F)$-conjugate to $(S, \theta \lambda)$ for some $\lambda \in X_{\mathrm{nr}}(S)$. This implies that there exists an $l \in L(F)$ such that $l^{-1} n \in$

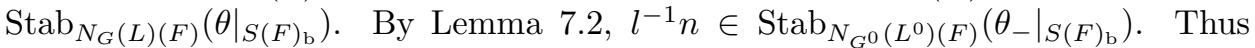
$l^{-1} n\left(S, \theta_{-}\right)=\left(S, \theta_{-} \lambda\right)$ and hence get a map $W^{\mathfrak{s}} \longrightarrow W^{\mathfrak{s}_{0}}$ induced by the map $n \in N^{\mathfrak{s}} \longrightarrow l^{-1} n \in N^{\mathfrak{s}_{0}}$. We claim that this map is an isomorphism. Suppose $n_{0} \in N^{\mathfrak{s}_{0}}$. Then $n_{0} \in N_{G^{0}}\left(L^{0}\right)(F)$ such that ${ }^{l_{0}^{-1} n_{0}}\left(S, \theta_{-}\right)=\left(S, \theta_{-} \lambda_{-}\right)$for some $l_{0} \in L^{0}(F)$ and some $\lambda_{-} \in X_{\mathrm{nr}}(S)$. Thus $l_{0}^{-1} n_{0} \in \operatorname{Stab}_{N_{G^{0}}(S)(F)}\left(\left.\theta_{-}\right|_{S(F)_{\mathrm{b}}}\right)$. By Lemma 7.2 $l_{0}^{-1} n_{0} \in \operatorname{Stab}_{N_{G}(S)(F)}\left(\left.\theta\right|_{S(F))_{\mathrm{b}}}\right)$. We now show that $l_{0}^{-1} n_{0} \in N_{G}(L)(F)$.

Recall that $Z\left(L^{0}\right) / Z(L)$ is $F$-anisotropic. That is,

$$
Z(L)_{\mathrm{s}}^{\circ}=Z\left(L^{0}\right)_{\mathrm{s}}^{\circ} .
$$

Let $n=l_{0}^{-1} n_{0}$. Since $n \in N_{G^{0}}\left(L^{0}\right)(F), n$ preserves $Z\left(L^{0}\right)$ and since $n$ is rational, $n$ preserves $Z\left(L^{0}\right)_{\mathrm{s}}^{\circ}$. Thus $n$ preserves $Z(L)_{\mathrm{s}}^{\circ}$ and therefore also preserves $Z_{G}\left(Z(L)_{\mathrm{s}}^{\circ}\right)=L$.

Finally since $\theta_{+}$is invariant under $l_{0}^{-1} n_{0},{ }_{0}^{l^{-1} n_{0}}(S, \theta)=\left(S, \theta \lambda_{-}\right)$. This completes the proof.

Theorem 7.4. There is an algebra isomorphism

$$
\mathfrak{Z}^{\mathfrak{F}}(G(F)) \cong \mathfrak{Z}^{\mathfrak{F}_{0}}\left(G^{0}(F)\right)
$$

Proof. By Theorem 7.1, the map

$$
\mathfrak{f}: \pi \otimes \nu \in \operatorname{Irr}^{\mathrm{t}}(L(F)) \mapsto \pi_{0} \otimes\left(\left.\nu\right|_{L^{0}(F)}\right) \in \operatorname{Irr}^{\mathrm{t}_{0}}\left(L^{0}(F)\right), \nu \in X_{\mathrm{nr}}(L(F))
$$

is an isomorphism of varieties and by Theorem 7.3 it is equivariant with respect to the isomorphism $\iota_{\pi}$. Consequently, there is an isomorphism of quotient varieties,

$$
\operatorname{Irr}^{\mathfrak{t}}(L(F)) / W^{\mathfrak{s}} \cong \operatorname{Irr}^{\mathfrak{t}_{0}}(L(F)) / W^{\mathfrak{s}_{0}} .
$$

Since $\mathfrak{Z}^{\mathfrak{s}}(G(F))$ (resp. $\mathfrak{Z}^{\mathfrak{s}_{0}}\left(G^{0}(F)\right)$ ) is the ring of regular functions on the Bernstein variety $\operatorname{Irr}^{\mathfrak{t}}(L(F)) / W^{\mathfrak{s}}\left(\operatorname{resp} . \operatorname{Irr}^{t_{0}}(L(F)) / W^{\mathfrak{s}_{0}}\right)$, the result follows. 


\section{HeCKE ALGEBRA OF TAME TYPES}

Let $G$ be tamely ramified. Consider the datum

$$
\Sigma=\left(\left(\vec{G}, M^{0}\right),(y,\{\iota\}), \vec{r},\left(K_{M^{0}}, \rho_{M^{0}}\right), \vec{\phi}\right)
$$

as in [17, §7.2]. So $\vec{G}=\left(G^{0}, \ldots, G^{d}\right)$ is a tamely ramified twisted Levi sequence in $G, M^{0}$ is a Levi subgroup of $G^{0}, \vec{r}=\left(r_{0}, \ldots, r_{d}\right)$ is a sequence of real numbers, $y$ is a point in $\mathcal{B}\left(M^{0}, F\right),\{\iota\}$ is a commutative diagram

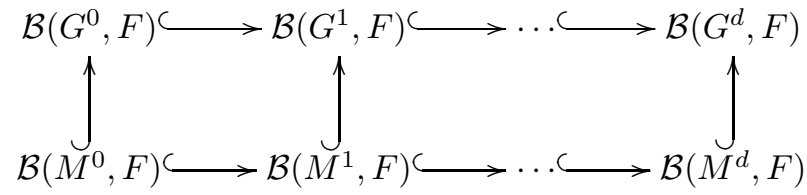

where $M^{i}$ is the centralizer in $G^{i}$ of $Z\left(M^{0}\right)_{\mathrm{s}}$. The diagram $\{\iota\}$ is required to be $\vec{s}$-generic relative to $y$ (see [17, $\S 3.5$, Definition]), where $\vec{s}=\left(0, r_{0} / 2, \ldots, r_{d-1} / 2\right)$. The group $K_{M^{0}}$ is a compact open subgroup of $M^{0}(F)$ containing the parahoric $M^{0}(F)_{y, 0}$ as a normal subgroup, and $\rho_{M^{0}}$ is an irreducible smooth representation of $K_{M^{0}}$ such that $\rho_{M^{0}} \mid M^{0}(F)_{y, 0}$ contains a cuspidal representation of the finite quotient $M^{0}(F)_{y, 0: 0+}$. Finally, $\vec{\phi}=\left(\phi_{0}, \ldots, \phi_{d}\right)$, where each $\phi_{i}$ is a character of $G^{i}(F)$. The datum $\Sigma$ is constrained by several conditions (loc. cit.).

From the datum $\Sigma$, Kim and Yu's construction produces a sequence of BushnellKutzko types $\left(K^{i}, \rho_{i}\right)$ for $G^{i}(F)$. Write $K=K^{d}$ and $\rho=\rho_{d}$.

Theorem 8.2. Let $\Sigma$ be the datum as above. Assume that for $0 \leq i \leq d, G^{i}$ is the Levi factor of a rational parabolic subgroup of $G$. Moreover, in the commutative diagram $\{\iota\}$, choose $\mathcal{B}\left(G^{i}, F\right) \hookrightarrow \mathcal{B}\left(G^{i+1}, F\right)$ to be $r_{i} / 2$-generic. Then $\Sigma$ determines an isomorphism

$$
\mathcal{H}\left(G, \rho^{\vee}\right) \stackrel{\sim}{\longrightarrow} \mathcal{H}\left(G^{0}, \rho_{0}^{\vee}\right) .
$$

Proof. Let $P=G^{d-1} U$ be a parabolic subgroup of $G$ with Levi factor $G^{d-1}$ and $\bar{P}=G^{d-1} \bar{U}$ the opposite parabolic. Then by [17, Lemma $\left.6.2(\mathrm{a})\right], K$ is decomposed with respect to $\left(U, G^{d-1}, \bar{U}\right)$. Let $K_{+}=K_{+}^{d}$ be as defined in [17, §7.4]. Then since $K \cap U=K_{+} \cap U$ and $K \cap \bar{U}=K_{+} \cap \bar{U}$ by the genericity of $\{\iota\}$, it follows that $K \cap U, K \cap \bar{U} \subset \operatorname{ker}\left(\rho_{d}\right)$. By 17, Theorem 8.1], the support of the Hecke algebra $\mathcal{H}\left(G, \rho^{\vee}\right)$ is contained in $K G^{d-1}(F) K$. Thus, by [7, Theorem 7.2(ii)],

$$
\mathcal{H}\left(G, \rho^{\vee}\right) \stackrel{\sim}{\longrightarrow} \mathcal{H}\left(G^{d-1}, \rho_{d-1}^{\vee}\right) .
$$

The result follows by induction.

Corollary 8.3. Let $\Sigma$ be as in equation 8.1). In the commutative diagram $\{\iota\}$, choose $\mathcal{B}\left(G^{i}, F\right) \hookrightarrow \mathcal{B}\left(G^{i+1}, F\right)$ to be $r_{i} / 2$-generic. Assume moreover that the connected center of $G^{0}$ is split modulo the center of $G$. Then $\Sigma$ determines an isomorphism

$$
\mathcal{H}\left(G, \rho^{\vee}\right) \stackrel{\sim}{\longrightarrow} \mathcal{H}\left(G^{0}, \rho_{0}^{\vee}\right) .
$$

Proof. Let $Z^{i}$ denote the connected center of $G^{i}, 0 \leq i \leq d$. Then $G^{i}$ is the centralizer of $Z^{i}$ in $G$. Since by assumption, $Z_{\text {an }}^{i}$ is contained in the center of $G$, it follows that $G^{i}$ is the centralizer of $Z_{\mathrm{s}}^{i}$ in $G$. Therefore all $G^{i}$ are in fact $F$-Levi subgroups. The result then follows from Theorem 8.2 . 
Assume $G$ is quasi-split and let $T$ be a maximal torus contained in an $F$-Borel subgroup. Write $T^{\text {der }}$ for the preimage of $T$ in $G^{\text {der }}$. Assume Hypothesis 5.1. Let $\theta$ be a character of $T(F)$. Let $(\vec{G}, \vec{\phi})$ denote the Kaletha-Howe factorization of $(T, \theta)$ and let $r_{i}$ denote the depth of $\phi_{i}$. Put $M^{0}=T, K_{M^{0}}=T(F)_{0}$ and $\rho_{M^{0}}=\theta \mid T(F)_{0}$. Since $T$ is contained in a Borel subgroup, $M^{i}=T$ for $0 \leq i \leq d$. Choose any point $y \in \mathcal{B}\left(M^{0}, F\right)$ and choose $\{\iota\}$ such that $\mathcal{B}\left(G^{i}, F\right) \hookrightarrow \mathcal{B}\left(G^{i+1}, F\right)$ is $r_{i} / 2$-generic. Let $\Sigma$ be the datum consisting of these choices and let $\left(K^{i}, \rho_{i}\right)$ be the Bushnell-Kutzko types for $G^{i}$ constructed out of $\Sigma$ by Kim and Yu's construction. Then $(K, \rho)$ is a $[T, \theta]_{G^{-}}$-type and $\left(K^{0}, \rho_{0}\right)$ is a $\left[T, \theta_{0}\right]_{G^{0}}$-type for a depth-zero character $\theta_{0}$ of $T(F)$.

Proposition 8.4. Assume that $p$ is co-prime to the order of $T_{\mathrm{an}} \cap T_{\mathrm{s}}$ and to the order of the component group $\pi_{0}\left(T_{\mathrm{an}} \cap T^{\mathrm{der}}\right)$. Suppose $\theta$ is a character of $T(F)$ that is trivial on $T_{\mathrm{an}}(F)_{0+}$. Let $\Sigma$ be a datum as in the previous paragraph. Then $\Sigma$ can be chosen suitably so that there is an isomorphism

$$
\mathcal{H}\left(G, \rho^{\vee}\right) \stackrel{\sim}{\longrightarrow} \mathcal{H}\left(G^{0}, \rho_{0}^{\vee}\right),
$$

determined by this choice.

Proof. From Theorem 8.2, it will be enough to show that the groups $G^{i}$ appearing in the datum $\Sigma$ are all Levi factors of parabolic $F$-subgroups of $G$. Equivalently, each $G^{i}$ is the centralizer in $G$ of an $F$-split torus.

We will see from Lemma 8.5 that $\theta$ has a Howe factorization $\left(\phi_{-1}, \phi_{0}, \ldots, \phi_{d}\right)$ where each character $\phi_{i}, i \geq 0$, is trivial on $T_{\text {an }}(F)$.

Choose an additive character $\Lambda$ of $F$ that is nontrivial on the ring of integers in $F$, but trivial on the prime ideal.

For $0 \leq i<d$, let $r_{i}$ denote the depth of $\phi_{i}$. Composing each $\left.\phi_{i}\right|_{T(F)_{r_{i}}}$ with the isomorphism $e_{i}: \operatorname{Lie}(T)(F)_{r_{i}} / \operatorname{Lie}(T)(F)_{r_{i}+} \longrightarrow T(F)_{r_{i}} / T(F)_{r_{i}+}$, we obtain a character of $\operatorname{Lie}(T)(F)_{r_{i}} / \operatorname{Lie}(T)(F)_{r_{i}+}$. Such a character must have the form $X \mapsto \Lambda(Y(X))$, where $Y \in \operatorname{Lie}^{*}(T)(F)_{-r_{i}} / \operatorname{Lie}^{*}(T)(F)_{-r_{i}+}$. We can choose a good coset representative $a_{i}$ in $Y$ so that $G^{i}$ is the centraliser $C_{G}\left(a_{i}\right)(F)$ of $a_{i}$ in $G$. By our assumption on $p$, we can in fact choose $a_{i}$ to be in $\operatorname{Lie}\left(T_{\mathrm{S}}\right)(F)$. Then it follows that $G^{i}$ is in fact an $F$-Levi subgroup. Indeed, $C_{G}\left(a_{i}\right)(F)=C_{G}\left(T_{a_{i}}\right)(F)$, where $T_{a_{i}}$ is the $F$-split subtorus of $T$ corresponding to the Galois fixed co-torsion-free submodule $X_{a_{i}}$ of the character lattice $X$ of $T$, where $X_{a_{i}}=\left\{x \in X \mid(d x)\left(a_{i}\right)=0\right\}$ where $d x$ denotes the derivative of $x$.

We take care of some unfinished business from the previous proof. Let $S \subseteq G$ be a tame maximal $F$-torus, and $S^{\prime} \subset S$ an $F$-subtorus. Denote by $S^{\text {der }}$ the preimage of $S$ in $G^{\text {der }}$. Assume that $p$ satisfies Hypothesis 5.1 and is co-prime to the order of the component group $\pi_{0}\left(S^{\prime} \cap S^{\text {der }}\right)$.

Lemma 8.5. Let $\theta: S(F) \longrightarrow \mathbb{C}^{\times}$be a character of depth $r>0$ that is trivial on $S^{\prime}(F)_{0+}$. Then the pair $(S, \theta)$ has a Howe factorization $\left(\phi_{-1}, \phi_{0}, \ldots, \phi_{d}\right)$, where each character $\phi_{i}, i \geq 0$, is also trivial on $S^{\prime}(F)$.

Proof. The existence of a Howe factorization is [15, Proposition 3.6.7]. For us, the key step is Lemma 3.6.9 loc. cit., which shows that if $\theta$ is $G$-generic, then there is a character $\phi$ of $G(F)$ such that $\phi$ and $\theta$ agree on $S(F)_{r}$. It will be enough to show that $\phi$ can be chosen to be trivial on $S^{\prime}(F)$.

The first part of the proof of Lemma 3.6.9 loc. cit. shows that $\theta$ is trivial on $S^{\mathrm{der}}(F)_{r}$. Write $D^{\prime}=G / S^{\prime} G^{\mathrm{der}}=S / S^{\prime} S^{\mathrm{der}}$. From Lemma 3.1.3 loc. cit., it 
follows that $D^{\prime}(F)_{r}=S(F)_{r} /\left(S^{\prime} S^{\text {der }}\right)(F)_{r}$. Now again by Lemma 3.1.3 loc. cit., $\left(S^{\prime} /\left(S^{\prime} \cap S^{\text {der }}\right)\right)(F)_{r} \cong\left(S^{\prime} /\left(S^{\prime} \cap S^{\text {der }}\right)^{\circ}\right)(F)_{r}=S^{\prime}(F)_{r} /\left(S^{\prime} \cap S^{\text {der }}\right)^{\circ}(F)_{r}$. Thus $\theta$ is trivial on $S^{\prime}(F)_{r}$ and on $\left(S^{\prime} /\left(S^{\prime} \cap S^{\text {der }}\right)\right)(F)_{r}=\left(S^{\prime} S^{\text {der }} / S^{\prime}\right)(F)_{r}$ and therefore on $\left(S^{\prime} S^{\text {der }}\right)(F)_{r}$. Consequently, $\theta$ descends to a character of $D^{\prime}(F)_{r}$. This character can be extended to a character $D^{\prime}(F) \rightarrow \mathbb{C}^{\times}$, trivial on $D^{\prime}(F)_{r+}$, which can then be pulled back to give a character $\phi$ of $G(F)$ that is trivial on $S^{\prime}(F)$, and whose restriction to $S(F)_{r}$ is equal to that of $\theta$.

Proposition 8.4 implies the following.

Corollary 8.6. Suppose the derived group $G^{\mathrm{der}}$ is F-split, and p satisfies Hypothesis 5.1. Then the Hecke algebra of every principal series block of $G(F)$ is isomorphic to the Hecke algebra of a depth-zero principal series block of a Levi subgroup $G^{0}(F)$ of $G(F)$ :

$$
\mathcal{H}\left(G, \rho^{\vee}\right) \stackrel{\sim}{\longrightarrow} \mathcal{H}\left(G^{0}, \rho_{0}^{\vee}\right) .
$$

Remark 8.7. The Hecke algebras of depth-zero Bernstein blocks are known due to the work of Morris [19]. Therefore Corollary 8.6 can be used to produce generators and relations for the Hecke algebras of principal series blocks of split groups. This was worked out by Roche 22. Note that Proposition 8.4 also applies to some principal-series blocks of non-split groups.

\section{Consequences for ABPS}

9.1. Twisted extended quotient. We recall here the notion of twisted extended quotients as given in 3 , §2.1]. Let $\Gamma$ be a group acting on a topological space $X$ and let $\Gamma_{x}$ denote the stabilizer in $\Gamma$ of $x \in X$. Let $\downarrow$ denote a family of 2-cocycles

$$
\natural_{x}: \Gamma_{x} \times \Gamma_{x} \longrightarrow \mathbb{C}^{\times} .
$$

Define

$$
\widetilde{X}_{\natural}=\left\{(x, \rho) \mid x \in X, \rho \in \operatorname{Irr} \mathbb{C}\left[\Gamma_{x}, \natural_{x}\right]\right\},
$$

where $\mathbb{C}\left[\Gamma_{x}, \natural_{x}\right]$ denotes the group algebra of $\Gamma_{x}$ twisted by $\natural_{x}$. Topologize $\widetilde{X}_{\natural}$ by requiring that a subset of $\widetilde{X}_{\natural}$ is open if and only if its projection to the first coordinate is open in $X$. Let $\left\{\phi_{\gamma, x} \mid(\gamma, x) \in \Gamma \times X\right\}$, denote a family of algebra isomorphisms

$$
\phi_{\gamma, x}: \mathbb{C}\left[\Gamma_{x}, \natural_{x}\right] \longrightarrow \mathbb{C}\left[\Gamma_{\gamma x}, \natural_{\gamma x}\right]
$$

satisfying the conditions:

(a) if $\gamma x=x$, then $\phi_{\gamma, x}$ is conjugation by an element of $\mathbb{C}\left[\Gamma_{x}, \natural_{x}\right]^{\times}$.

(b) $\phi_{\gamma^{\prime}, \gamma x} \phi_{\gamma, x}=\phi_{\gamma^{\prime} \gamma, x}$ for all $\gamma^{\prime}, \gamma \in \Gamma$ and $x \in X$.

Define a $\Gamma$-action on $\widetilde{X}_{\natural}$ by

$$
\gamma \cdot(x, \rho)=\left(\gamma x, \rho \circ \phi_{\gamma, x}^{-1}\right) .
$$

Then the twisted extended quotient of $X$ by $\Gamma$ is defined to be:

$$
(X / / \Gamma)_{\natural}:=\tilde{X}_{\natural} / / \Gamma \text {. }
$$

Remark 9.1. The twisted extended quotient depends on the choices of the algebra isomorphisms $\phi_{\gamma, x}$. If $\natural_{x}$ is trivial for all $x \in X$, then there is a canonical choice. Namely, $\phi_{\gamma, x}$ is conjugation by $\gamma$ (see $[\underline{3}, \S 2.1]$ ). 
9.2. ABPS Conjecture. Write $\mathfrak{s}=[L, \pi]_{G}, \mathfrak{t}=[L, \pi]_{L}$ and let $W^{\mathfrak{s}, t}$ denote the stabilizer in $W^{\mathfrak{s}}$ of a point $t$ in $\operatorname{Irr}^{\mathfrak{t}}(L(F))$.

The ABPS conjecture [3, §2.3] asserts that there exists a family of 2-cocycles

$$
\natural_{t}: W^{\mathfrak{s}, t} \times W^{\mathfrak{s}, t} \longrightarrow \mathbb{C}^{\times}, t \in \operatorname{Irr}^{\mathfrak{t}}(L(F))
$$

such that there is a natural bijection

$$
\operatorname{Irr}^{\mathfrak{s}}(G(F)) \longleftrightarrow\left(\operatorname{Irr}^{\mathfrak{t}}(L(F)) / / W^{\mathfrak{s}}\right)_{\natural} .
$$

This bijection is expected to be compatible with the local Langlands correspondence (loc. cit.). If $G$ is quasi-split, the cocycles in the family $\downarrow$ are expected to be trivial.

9.3. An isomorphism for extended quotients. Let $\mathfrak{s}_{0}$ and $\mathfrak{t}_{0}$ be as in $\$ 7$ Let $\iota_{\pi}$ be as in Theorem 7.3 and $\mathfrak{f}$ as in Theorem 7.1. Then for $t \in \operatorname{Irr}^{\mathfrak{t}}(L(F))$, since $\mathfrak{f}$ is equivariant with respect to $\iota_{\pi}$ by Theorem 7.3 , we have

$$
\left.\iota_{\pi}\right|_{W^{\mathfrak{s}, t}}: W^{\mathfrak{s}, t} \stackrel{\sim}{\longrightarrow} W^{\mathfrak{s}_{0}, \mathfrak{f}(t)} .
$$

Each 2-cocycle $\natural_{t}, t \in \operatorname{Irr}^{\mathrm{t}}(L(F))$, therefore defines a 2-cocycle

$$
\natural_{\mathfrak{f}(t)}^{0}: W^{\mathfrak{s}_{0}, \mathfrak{f}(t)} \times W^{\mathfrak{s}_{0}, \mathfrak{f}(t)} \longrightarrow \mathbb{C}^{\times} .
$$

The following theorem is then immediate from Theorem 7.3 and [18, Theorem 6.1].

Theorem 9.3. Suppose Hypothesis 5.1. Then there is an isomorphism

$$
\mathfrak{l}_{\pi}:\left(\operatorname{Irr}^{\mathfrak{t}}(L(F)) / / W^{\mathfrak{s}}\right)_{\mathfrak{\natural}} \stackrel{\sim}{\longrightarrow}\left(\operatorname{Irr}^{\mathfrak{t}_{0}}\left(L^{0}(F)\right) / / W^{\mathfrak{s}_{0}}\right)_{\mathfrak{\natural}^{0}}
$$

determined by the choice of $(S, \theta)$.

Let $(K, \rho)$ (resp. $\left(K^{0}, \rho_{0}\right)$ be the type constructed by Kim and Yu for $\mathcal{R}^{\mathfrak{s}}(G(F))$ (resp. $\mathcal{R}^{\mathfrak{s}_{0}}\left(G^{0}(F)\right)$ ). We have equivalences of categories:

$$
\mathcal{R}^{\mathfrak{s}}(G(F)) \stackrel{\sim}{\longrightarrow} \mathcal{H}\left(G, \rho^{\vee}\right)-\operatorname{Mod}, \quad \mathcal{R}^{\mathfrak{s}_{0}}\left(G^{0}(F)\right) \stackrel{\sim}{\longrightarrow} \mathcal{H}\left(G^{0}, \rho_{0}^{\vee}\right)-\text { Mod. }
$$

Corollary 9.4. Suppose Hypothesis 5.1, Then for regular Bernstein blocks, Conjecture 1.1 implies the ABPS Conjecture.

Proof. For depth-zero Bernstein blocks, the ABPS conjecture holds by [25] and Morris's presentation of Hecke algebras of depth-zero blocks [19]. The result then follows from Theorem 9.3 .

Theorem 9.5. Assume Hypothesis 5.1. Assume that the twisted Levi subgroups $G^{0} \subsetneq \ldots \subsetneq G^{d}=G$ obtained in the Howe factorization of $(S, \theta)$ in $G$ are F-Levi subgroups of $G$. Then the ABPS conjecture holds for the Bernstein block $\mathcal{R}^{\mathfrak{s}}(G(F))$. In particular, if the connected center of $G^{0}$ is split modulo the center of $G$, then the ABPS Conjecture holds. Therefore it also holds for principal series blocks of split groups.

Proof. The first claim follows from Theorem 8.2 and Corollary 9.4 , The last two claims follow from Corollaries 8.3, 8.6 and 9.4.

Remark 9.6. When $G$ is a classical group, Equation (1.3) for certain Bernstein blocks can be observed from results of Kim [16. More generally, for classical groups, Heiermann [12] has established certain equivalences of categories in the spirit of Equation (1.4). It would be worthwhile to understand the equivalence in terms of Kim-Yu data. 


\section{Partial Results toward FURTher HeCKe Algebra isomorphisms}

We prove weaker versions of the Hecke algebra isomorphism of Equation (1.3). Let the notation be as in $\$ 7$ and $\$ 9.3$.

The equivalence

$$
\mathcal{R}^{\mathfrak{s}}(G(F)) \stackrel{\sim}{\longrightarrow} \mathcal{H}\left(G, \rho^{\vee}\right)-\operatorname{Mod},
$$

induces an isomorphism of $\mathfrak{Z}^{\mathfrak{s}}$ with the center of $\mathcal{H}\left(G, \rho^{\vee}\right)$. View $\mathcal{H}\left(G, \rho^{\vee}\right)$ as a $\mathfrak{Z}^{\mathfrak{s}}$-algebra. It is then finitely generated as a $\mathfrak{Z}^{\mathfrak{s}}$-module. Similarly, $\mathcal{H}\left(G^{0}, \rho_{0}^{\vee}\right)$ is a finitely generated module over its center $\mathfrak{Z}^{\mathfrak{s}_{0}}$.

Write $S=\mathfrak{Z}^{\mathfrak{s}} \backslash 0$ (resp. $S_{0}=\mathfrak{Z}^{\mathfrak{s}_{0}} \backslash 0$ ) and let $\mathrm{k}_{\mathfrak{s}}:=S^{-1} \mathfrak{Z}^{\mathfrak{s}}\left(\right.$ resp. $\left.\mathrm{k}_{\mathfrak{s}_{0}}:=S_{0}^{-1} \mathfrak{Z}^{\mathfrak{s}_{0}}\right)$ denote the field of fractions of $\mathfrak{Z}^{\mathfrak{s}}$ (resp. $\left.\mathfrak{Z}^{\mathfrak{s} 0}\right)$. Write

$$
\mathrm{H}\left(G, \rho^{\vee}\right):=S^{-1} \mathcal{H}\left(G, \rho^{\vee}\right) \text {. }
$$

Similarly,

$$
\mathrm{H}\left(G^{0}, \rho_{0}^{\vee}\right):=S_{0}^{-1} \mathcal{H}\left(G^{0}, \rho_{0}^{\vee}\right) .
$$

In 6. §4.3], Bushnell and Henniart define the notions of generic and simply generic, which are generalizations of the generic representations in quasi-split groups.

Theorem 10.1. Assume Hypothesis 5.1. Assume that $\left.\pi\right|_{{ }^{\circ} L(F)}$ and $\left.\pi_{0}\right|_{\circ} L^{0}(F)$ are multiplicity free. Then there is a vector-space isomorphism

$$
\mathcal{H}\left(G, \rho^{\vee}\right) \stackrel{\sim}{\longrightarrow} \mathcal{H}\left(G^{0}, \rho_{0}^{\vee}\right) .
$$

If $\mathfrak{s}$ and $\mathfrak{s}_{0}$ are simply generic, then there is a $\mathbb{C}$-algebra isomorphism

$$
\mathrm{H}\left(G, \rho^{\vee}\right) \stackrel{\sim}{\longrightarrow} \mathrm{H}\left(G^{0}, \rho_{0}^{\vee}\right) .
$$

Proof. By [23, Prop. 1.8.4.1], $\mathcal{H}\left(G, \rho^{\vee}\right)$ (resp. $\left.\mathcal{H}\left(G^{0}, \rho_{0}^{\vee}\right)\right)$ is a free right-module over $\mathfrak{Z}^{\mathfrak{t}}\left(\operatorname{resp} . \mathfrak{Z}^{\mathfrak{t}_{0}}\right)$ of rank $\left|W^{\mathfrak{s}}\right|\left(\right.$ resp. $\left.\left|W^{\mathfrak{s}_{0}}\right|\right)$. By [18, Theorem 6.1], $\mathfrak{Z}^{\mathfrak{t}} \cong \mathfrak{Z}^{\mathfrak{t}_{0}}$ and by Theorem $7.3 . W^{\mathfrak{s}} \cong W^{\mathfrak{s} 0}$. The first claim is then immediate.

By [6. Theorem 5.2],

$$
\mathrm{H}\left(G, \rho^{\vee}\right) \cong \mathrm{M}_{n}\left(\mathrm{k}_{\mathfrak{s}}\right),
$$

for some integer $n$. Similarly

$$
\mathrm{H}\left(G^{0}, \rho_{0}^{\vee}\right) \cong \mathrm{M}_{n_{0}}\left(\mathrm{k}_{\mathfrak{s}_{0}}\right),
$$

for some integer $n_{0}$. Since $\mathrm{k}_{\mathfrak{s}} \cong \mathrm{k}_{\mathfrak{s}_{0}}$ by Theorem 7.4 , to prove $\mathrm{H}\left(G, \rho^{\vee}\right) \stackrel{\sim}{\rightarrow} \mathrm{H}\left(G^{0}, \rho_{0}^{\vee}\right)$, it suffices to prove

$$
\operatorname{dim}_{\mathrm{k}_{\mathfrak{s}}} \mathrm{H}\left(G, \rho^{\vee}\right)=\operatorname{dim}_{\mathrm{k}_{\mathfrak{s}_{0}}} \mathrm{H}\left(G^{0}, \rho_{0}^{\vee}\right) .
$$

Now $S^{-1} \mathfrak{Z}^{\mathrm{t}} \cong S_{0}^{-1} \mathfrak{Z}^{\mathrm{t}_{0}}$ by Theorem 7.4 and [18, Theorem 6.1]. The claim then follows from [23, Prop. 1.8.4.1].

\section{REFERENCES}

[1] Jeffrey D. Adler and Alan Roche, An intertwining result for p-adic groups, Canad. J. Math. 52 (2000), no. 3, 449-467. MR1758228 (2001m:22032)

[2] Jeffrey D. Adler and Dipendra Prasad, Decomposition upon restriction to the derived subgroup, Pacific J. Math 300 (2019), 1-14, available at arXiv:1806.03635

[3] Anne-Marie Aubert, Paul Baum, Roger Plymen, and Maarten Solleveld, Conjectures about $p$-adic groups and their noncommutative geometry, Around Langlands correspondences, Contemp. Math., vol. 691, Amer. Math. Soc., 2017, pp. 15-51. MR3666049

[4] - The principal series of p-adic groups with disconnected center, Proc. Lond. Math. Soc. (3) 114 (2017), no. 5, 798-854, DOI 10.1112/plms.12023.

[5] J. N. Bernstein, Le "centre" de Bernstein, Representations of reductive groups over a local field, Travaux en Cours, Hermann, Paris, 1984, pp. 1-32. MR771671 (86e:22028) 
[6] Colin J. Bushnell and Guy Henniart, Generalized Whittaker models and the Bernstein center, Amer. J. Math. 125 (2003), no. 3, 513-547.

[7] Colin J. Bushnell and Philip C. Kutzko, Smooth representations of reductive p-adic groups: structure theory via types, Proc. London Math. Soc. (3) 77 (1998), no. 3, 582-634, DOI 10.1112/S0024611598000574. MR1643417

[8] Kwangho Choiy, On multiplicity in restriction of tempered representations of p-adic groups, Math. Z. 291 (February 2019), no. 1-2, 449-471, available at arXiv:1306.6118

[9] Jessica Fintzen, Types for tame p-adic groups, Ann. Math., to appear, available at arXiv: 1810.04198

[10] , Tame cuspidal representations in non-defining characteristics (May 15, 2019), available at arXiv: 1905.06374

[11] Jeffrey Hakim and Fiona Murnaghan, Distinguished tame supercuspidal representations, Int. Math. Res. Pap. IMRP 2 (2008), Art. ID rpn005, 166. MR2431732

[12] Volker Heiermann, Local Langlands correspondence for classical groups and affine Hecke algebras, Math. Z. 287 (2017), no. 3-4, 1029-1052, DOI 10.1007/s00209-017-1858-3.

[13] — Opérateurs d'entrelacement et algèbres de Hecke avec paramètres d'un groupe réductif p-adique: le cas des groupes classiques, Selecta Math. (N.S.) 17 (2011), no. 3, 713756, DOI 10.1007/s00029-011-0056-0.

[14] Roger E. Howe, Harish-Chandra homomorphisms for p-adic groups, with the collaboration of Allen Moy, CBMS Regional Conference Series in Mathematics, vol. 59, Published for the Conference Board of the Mathematical Sciences, Washington, DC, 1985. MR821216 (87h:22023)

[15] Tasho Kaletha, Regular supercuspidal representations, J. Amer. Math. Soc., published electronically July 18, 2019, DOI 10.1090/jams/925, available at arXiv: 1602.03144

[16] Ju-Lee Kim, Hecke algebras of classical groups over p-adic fields. II, Compositio Math. 127 (2001), no. 2, 117-167, DOI 10.1023/A:1012023315726.

[17] Ju-Lee Kim and Jiu-Kang Yu, Construction of tame types, Representation theory, number theory, and invariant theory, 2017, pp. 337-357.

[18] Manish Mishra, Bernstein center of supercuspidal blocks, J. Reine Angew. Math. 748 (2019), 297-304, DOI 10.1515/crelle-2016-0041. MR3918438

[19] Lawrence Morris, Tamely ramified intertwining algebras, Invent. Math. 114 (1993), no. 1, 1-54, DOI 10.1007/BF01232662.

[20] Allen Moy, Representations of $\mathrm{U}(2,1)$ over a p-adic field, J. Reine Angew. Math. 372 (1986), 178-208. MR863523 (88a:22031)

[21] - Representations of $G \mathrm{Sp}(4)$ over a p-adic field. I, II, Compositio Math. 66 (1988), no. 3, 237-284, 285-328. MR948308 (90d:22022)

[22] Alan Roche, Types and Hecke algebras for principal series representations of split reductive p-adic groups, Ann. Sci. École Norm. Sup. (4) 31 (1998), no. 3, 361-413, DOI 10.1016/S00129593(98)80139-0.

[23] _ The Bernstein decomposition and the Bernstein centre, Ottawa lectures on admissible representations of reductive $p$-adic groups, Fields Inst. Monogr., vol. 26, Amer. Math. Soc., Providence, RI, 2009, pp. 3-52. MR2508719

[24] Maarten Solleveld, Endomorphism algebras and Hecke algebras for reductive p-adic groups (2020), available at arXiv:2005.07899

[25] On the classification of irreducible representations of affine Hecke algebras with unequal parameters, Represent. Theory 16 (2012), 1-87, DOI 10.1090/S1088-4165-2012-00406$\mathrm{X}$.

[26] Jiu-Kang Yu, Construction of tame supercuspidal representations, J. Amer. Math. Soc. 14 (2001), no. 3, 579-622, DOI 10.1090/S0894-0347-01-00363-0. MR1824988

Department of Mathematics and Statistics, American University, 4400 Massachusetts Ave NW, Washington, DC 20016-8050, USA

Email address: jadler@american.edu

Department of Mathematics, Indian Institute for Science Education and Research,

Dr. Homi Bhabha Road, Pashan, Pune 411 008, India

Email address: manish@iiserpune.ac.in 